\title{
IX.
}

\section{Verschluss des linken ostium arteriosum in dem Herzen eines halbjährigen Kindes.}

\author{
Untersucht und beschrieben \\ yon \\ Professor Bardeleben. \\ Hierzu Tafel III.
}

\begin{abstract}
Durch die Gefälligkeit des Herrn Professor Wernher erhielt ich zur anatomischen Untersuchung ein an denselben, als Director der pathologischen Sammlung zu Giefsen, von einem praktischen Arzte übersandtes Herz cum annexis, welches mir, seiner höchst eigenthïmlichen Mifsbildung wegen, einer besonderen Beschrcibung werth zu sein scheint. Ich schicke das den Sektionsbericht enthaltende Schreiben des Arztes, soweit es hierher gehört, so wie auch die Angaben über die an dem Kinde, aus welchem das Herz herrührt, während des Lebens gemachten Beobachtungen voraus, um mich darauf später beziehen zu können. Leider muls man darin Vieles vermissen.
\end{abstract}

Sektionsbericht des behandelnden Arztes.

„Bei der Oeffnung des Bauches nahm die Leber den grölse„ren Theil der Bauchhöhle ein, und war mil schwarzem Blute "sehr überfüllt. Sonst Nichts Abnormes in der Bauchhöhle. „Nach Eröffnung des Brustkastens fand sich ein ziemlich 
"grofses Herz. Die Lungen waren sehr blutleer. Ich schnitt „die art. aoria dort wo sie als aorta descendens erscheint „ab, konnte aber die grofsen aufsteigenden Gefälse, welche in "diesem Zwischenraume ihren Ursprung nehmen, nicht ge"wahren. An der absteigenden aorta gewahrte ich einige "aufsteigende Aeste."

Lebens- und Krankheitsgeschichte des Kindes.

"Vor der Geburt, selbst in den letzten Tagen machte das „Kind sehr schwache kaum fühlbare Bewegungen. Nach der „Geburt zeigte es ein schwaches Leben, lag halbe Tage bei"nahe ohne eine Bewegung, ohne einen Laut von sich zu geben. „Wenn es die Brust nehmen sollte, so zog es zwei- auch "dreimal, und fing dann an zu weinen und zu husten, als „wenn ihm zum Saugen die Kraft und der Athem fehlte, so "dafs es nur mit gröIster Mühe ernährt werden konnte. Als "es 3 Wochen alt war, bekam es über den ganzen Körper „einen starken Ausschlag, so dafs sich die Haut fast ganz ab„schälte, und man es beinah gar nicht anfassen konnte. Dar„auf wurden die Haut, der Mund und die Nägel blau und „blieben es auch bis zum Tode des Kindes. Selten schlief „es unausgesetzt länger als 2 Stunden, schrie dann plötzlich "laut auf, zappelte mit den Händen und zog die Fülse krampf"haft nach hinten; dann wurden der Mund, die Zunge und "die Nägel beinahe dunkelblau, und es war nur dadurch zur „Ruhe zu bringen, dafs man es auf die linke Seite legte und "heftig wiegte. Solche Anfälle wiederholten sich besonders „dann häufiger, wenn der Stublgang nicht regelmäfsig war. „Dieser erfolgte aber gewöhnlich vier- bis fünfmal des Tages. „So lebte das Kind beinahe 27 Wochen, als es (4 Tage vor „seinem Tode) sich zu erholen schien, munterer wurde und ,häufig lachte. 2 Tage darauf fing es in der Nacht plötzlich "an heftig zu schreien, die oben erwähnten Zufälle wieder"holten sich häufiger und heftiger, bis es endlich am Mittag. "des vierten Tages sanft entschlief." - 
Ich lasse nun die Resultate meiner anatomischen Untersuchung folgen. -

Bei äufserer Betrachtung erschien das Herz selbst ziemlich wohlgebildet, nur die auricula dextra etwas zu grols, die sinistra etwas zu klein. Die beiden Hohlvenen, die obere dicht ïber dem rechten Vorhof abgeschnitlen, und die Lungenvenen, deren eine abgerissen war, boten nichts $A b$ weichendes dar. Dagegen schien eine Aorta ganz zu fehlen, während die art. pulmonalis, sehr viel stärker als gewöhnlich, nach Abgabe ihrer beiden Lungenäste, sich als ein sehr weiter Ductus arterios. Botalli fortsetzte. Leider war aber der Schnitt hier so dicht an der Abgangsstelle der Lungenäste geführt, dafs über das Verhältnils dieses grofsen Duct. art. zur Aorta descend. und zu den sonst aus dem Arcus aortae entspringenden Arterien nur durch Schliusse aus anderweitig bekannten. Thatsachen einige Klarheit erlangt werden kann. Genauere Präparation der art. pulmonalis liefs mich alsbald neben ihr und zwar an ihrer rechten Seite und etwas nach hinten, ihr dicht anliegend, einen Canal entdecken, dessen weitere Verfolgung mich zu den Herzkranzarterien fuhrte, und aus dessen hinterem Umfange aulserdem ein Paar sehr dünne Aestchen ihren Ursprung nahmen. Zwischen den beiden Herzkranzarterien setzt sich von diesem Canale gegen die linke Herahälfte hin, hinter der art. pulmonalis, ein dünner solider Strang fort. Dieser Strang ist das krankhaft verschlossene Stiuck der Aorta, der Canal dasjenige Stïck derselben, durch welches das Blut aus der cort. descend. rückwärts strömen mufste, um in die art. coronariae zu gelangen. Was sonst aort. ascendens und arcus aortae ist, war hier nichts als der Stamm der beiden art coronariae, mit dem sie aus der aort. desc, entsprangen. -

Das Innere des Herzens zeigte Folgendes. Beide Herzhöhlen der rechten Seite sind beträchtlich erweitert. Im rechten Vorhof sind überaus starke musculi pectinati entwickelt. Die Mlindung der Herzkranzvene ist normal. Die valvula Eustachii ist grofs, das tuberculum Loweri undeut- 
lich. Die valvula foraminis ovalis ist stark nach rechts gewölbt und verschliefst das eirunde Loch bei weitem nicht vollständig. Am vorderen oberen Umfange desselben befindet sich eine weit offen stehende Lücke von dem Durchmesser einer Rabenfeder. Die valvula tricuspidalis ist stark und muskulös, derjenigen des Vogelherzens ähnlich. Die halbmondförmigen Klappen am rechten ostium arteriosum sind auffallend stark, mit sehr deullichen Knölchen versehen und schliefsen sehr vollkommen. Der linke Vorhof ist etwa den vierten Theil so grofs als der rechte, das ostium venosum des linken Ventrikels sehr eng, so dafs es von dem geöffneten Vorhofe aus schwer entdeckt werden konnte. Der linke Ventrikel selbst ist reducirt auf eine kleine nach unten zweizipflige Höhle von nur $17 \mathrm{Mm}$. Höhe und einem Queerdurchmesser von $9 \mathrm{Mm}$., im grölsten Umfange gemessen. Die muskulösen Wandungen dieses Ventrikelrudiments haben an den dünnsten Stellen $10 \mathrm{Mm}$., an den dicksten aber $20 \mathrm{Mm}$. Durchmesser, während derselbe an der rechten Herzkammer im maximo nur $9 \mathrm{Mm}$, an den dünnsten Stellen derselben aber nur $6 \mathrm{Mm}$. beträgt. Die valvula mitralis ist, der Grölse des Ventrikels entsprechend, regelmälsig entwickelt. Die innere Auskleidung der Kammer durchaus glatt und normal. Da wo das ostium arteriosum sich befinden sollte, kann man eine kleine Strecke weit eine dünne Schweinsborste in einen blind endigenden Canal einschieben. Irgend ein Zusammenhang mit der Aorta findet sich nicht.

Vorstehende anatomische Beschreibung wird durch die beigefügten Abbildungen ihre nähere Erläuterung finden. Ich verdanke dieselben der geschickten Hand des Herru $\boldsymbol{D r}$. med. W. Hillebrand, meines ehemaligen Zuhörers, dem ich den herzlichsten Dank dafür hiermit öffentlich ausspreche.

Der Mechanismus des Kreislaufs in diesem milsgebildeten Herzen kann wohl nur folgender gewesen sein:

Das durch die Hohlvenen in den rechten Vorhof einströmende venöse Blut wurde durch dessen Zusammeuziehung in den rechten Ventrikel getrieben. Der Eintrilt in den linken 
Vorhof durch das weit offenstehende foramen ovale war unmöglich; im Gegentheil mulste fort und fort das aus den Lungen durch die venae pulmonales in den linken Vorhof einströmende Blut, nachdem es zum sehr geringen Theil in den blind endigenden kleinen linken Ventrikel eingetrieben und aus ihm regurgitirt war, durch das fortemen ovale seinen $W_{\text {eg }}$ in den rechten Vorhof nehmen und mit dem Hohlvenenblute gemischt in den rechten Ventrikel gelangen. Der linke Vorhof und die linke Herzkammer waren also ihrer Function nach blofs als Divertikel der rechten Vorkammer zu betrachten. Functionell gab es in diesem Herzen nur eine Kammer, die rechte. Aus dieser strömte das gemischte Blut in die ehemalige linke Aorta (Art. pulmonalis), aus welcher zwei Seitenäste einen Theil desselben zu den Lungen führten, während die grölsere Masse ihren Weg durch die weitere Fortsetzung dieser linken Aorta (Ductus art. Botall.) zur Aorta descendens fortsetzte. Unzweifelhaft entsprang nun hier an der Stelle wo normaler Weise die beiden Aorten zusammenstofsen, der gemeinsame Stamm der Arteriae coronariae cordis (die degradirte eigentliche Aorta), in welchem das Blut nach dem Herzen zurückströmte zu dessen Ernährung. Wahrscheinlich ist aus der oben angeführten Angabe des secirenden Arztes, dafs die Stämme für die obere Körperhälfte erst aus der Aorta descendens entsprangen. Wären die Brusteingeweide lege artis herausgenommen worden, so hätte man leicht über diesen interessanten Punkt Aufschlufs erhalten können.

Es liegt sehr nahe dies Herz mit einem Amphibienherzen zu vergleichen, wenn man die functionelle Bedeutung seiner einzelnen Theile beriicksichligt; denn es bestand in der That nur aus einer Vorhofs- und einer Ventrikelhöhle; in erstere flofs venöses und arterielles Blut zusammen; aus letzterer erhielt nicht blofs der ganze Körper, sondern auch das Athemorgan sein Blut; die Lungenblutbahn war eine Fraclion der Körperblutbahn; nur ein kleiner Theil der Blutmasse ath mete. Durch einen solchen Vergleich könnte nun auch der Gedanke an eine Bildungshemmung als Ursache dieser Mifs- 
bildung nahe gelegt werden, Dies muls aber durchaus zurückgewiesen werden. Wir können wohl mit Bestimmtheil behaupten, dafs die Veranlassung eine adhäsive Entzündung des ostium arteriosum des linken Ventrikels war, welche sich zu einer Zeit entwickelte, wo alle einzelnen Theile des Herzens schon in bleibender Weise fertig gebildet, aber noch sehr klein waren; vielleicht im dritten Monate. Durch eine solche Annahme werden alle Abweichungen vom Normalzustande leicht und vollständig erklärt. Selbst die enorme Hypertrophie der Wandungen des linken Ventrikels liefse sich als Folge der bis zum Verschluls gesteigerten Stenose des ostium arteriosum begreifen. Nur der tiefe Ursprung der Aeste für die obere Körperhälfte (wenn er wirklich Statt fand) würde immer für eine davon unabhängige Varietät erklärt werden müssen. Wodurch aber die von mir angenommene Entzündung (lokale Arteriits) hervorgerufen wurde, daruber etwas Bestimmtes auszusagen, möchte wohl zu dem schwierigsten Theile der schwierigen Aeliologie der Krankheiten des Foetus gehören. Endlich liegt wohl am Tage, dafs ein mit solchem Amphibienherzen behaftetes Individuum auf die Dauer nicht lebensfähig sein konnte; es ist vielmehr zu verwundern, dafs es so lange gelebt hat. Im Interesse der Wissenschaft muls bedauert werden, dafs ïber die Temperatur des Kindes keine Beobachtungen angestellt und die Auskultation nicht vorgenommen wurde; beide hälten gewifs interessante Resultate geliefert. - Von den in der oben mitgetheilten Krankheitsgeschichte aufgeführten Symptomen ergiebt sich die Cyanose wohl als die nothwendige Folge des "Amphibienherzens". Trat sie in den ersten 3 Wochen nicht hervor, weil die in der ersten Zeit des Extrauterinallebens durch die dünnere und feuchtere Epidermis hindurch leichter Statt findende Hautrespiration die mangelhafte Oxydation des Blules in den Lungen vollständiger ersetzte? oder kam die venöse Stase erst allmälig zu Stande? Eine bestimmte Antwort möchte wohl nach den bisherigen Untersuchungen über Cyanose überhaupt unmöglich sein. - Die Trägheit und Kraftlosigkeit der Bewe- 
gungen erklärt sich hier, wie in allen ähnlichen Fällen aus der unvollkommenen Ernährung der Muskeln und Nerven durch das fast ganz venöse Blut; die Athemnolh beim Saugen aber findet, abgesehen hiervon, noch darin ihre Begründung, dafs die qualitativ so wenig wirksame Function der Lungen gewifs keinen Augenblick unterbrochen werden durfte, ohne sogleich die Gefahr vollständiger Asphyxie herbeizuführen. -

\section{Erklärung der Abbildungen. (s. Tafel III.)}

Fig. 1. Ansicht des Präparats von vorn.

L. Leber.

$F$. $F$. Lunge.

T. Trachea.

V. vena cava inferior.

C. vena cava superior.

a. atrium dextrum.

b. atrium sinistrum.

P. arteria mulmonalis.

B. Fortsetzung derselben zum Aortenbogen (Ducl. art. Botalli).

$\boldsymbol{p}$. $\boldsymbol{p}$. Aeste der $\boldsymbol{a}$. pulnionalis zu den Lungen.

Die punktirten Linien deuten die bestimmt roranszusetzenden Verbindung der Aorta ascend. mit dem Duct. art. Botall. zur Aortn desc. an. Die Stelle, wo die Aeste für die obere Körperhälfte entsprungen sein möchten, ist nicht angedentet.

Fig. 2. Ansicht von oben.

Die Leber ist nach links,

die Lungen nach rechts gelegt,

Lungenarterie nnd obere Hollader sind auseinander gezogen.

M. aufgeschnittene Aorta mit den aus ihr entspringenden art. coronariae.

R. solider Strang (obliterirtes Stïck der Aorta.)

x. y. kleine Arterine medinstini.

$r$. auricula dextrn.

s. auricula sinistra.

t. hintere Wand der Vorhöfe. Die übrigen Buclstaben wie in Fignr 1.

Fig. 3. Ansicht von der rechten Seite.

Der rechte Yorhof ist geöffnet. 


\section{2}
a. auricula dextra.
o. formen ovale.
7. dessen Klappe.
$\boldsymbol{H}$. rechte Herzkanmer.
E. valvula Eustachii.
Das Uebrige wie in Figur 1.

Fig. 4. Linke Herzhälfte, geöffuet.

$v$. Die von dicken Muskelwandungen umgebene rudimentäre Höhle des linken Yentrikels.

A. Iinkes Atrinm.

S. dẹssen Auricula.

l. rudimentäre valvula mitralis, deren einer Zipfel durch den Schnitt gespalten ist.
o. forramen ovale.
k. dessen valvala.
p. eine vena pulnonalis.
P. die abgeschnittene Art. pulm. 

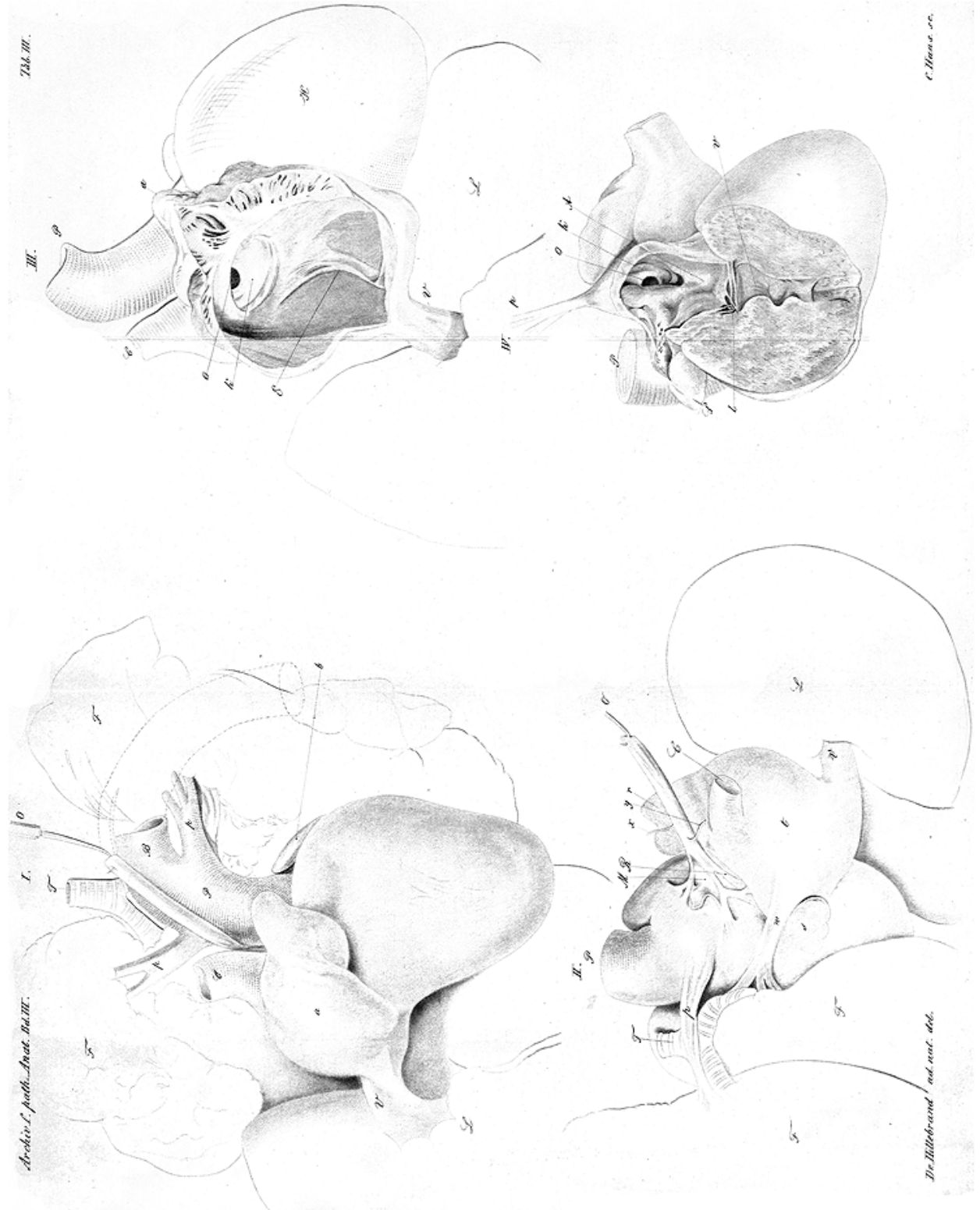